\title{
ECTO-MESENCHYMAL STEM CELLS FROM DENTAL PULP ARE COMMITTED TO DIFFERENTIATE INTO ACTIVE MELANOCYTES
}

\author{
Francesca Paino ${ }^{1}$, Giulia Ricci ${ }^{1,2}$, Alfredo De Rosa ${ }^{3}$, Riccardo D’Aquino ${ }^{1,3}$, Luigi Laino ${ }^{3}$, Giuseppe Pirozzi ${ }^{4}$, \\ Virginia Tirino*, and Gianpaolo Papaccio*
}

\author{
${ }^{1}$ Department of Experimental Medicine, Histology and Embryology, TERM Division, School of Medicine, Second \\ University of Naples \\ ${ }^{2}$ National Institute of Biostructures and Biosystems Interuniversitary Consortium - Unit of Sections of Naples \\ ${ }^{3}$ Department of Odontostomatology, School of Medicine, Second University of Naples \\ ${ }^{4}$ Department of Experimental Oncology, National Cancer Institute, Naples, Italy
}

\begin{abstract}
Dental pulp stem cells (DPSCs) are multipotent stem cells derived from neural crest and mesenchyme and have the capacity to differentiate into multiple cell lineages. It has already been demonstrated that DPSCs differentiate into melanocyte-like cells but only when cultivated in a specific melanocyte differentiating medium. In this study we have shown, for the first time, that DPSCs are capable of spontaneously differentiating into mature melanocytes, which display molecular and ultrastructural features of full development, including the expression of melanocyte specific markers and the presence of melanosomes up to the terminal stage of maturation. We have also compared the differentiating features of DPSCs grown in different culture conditions, following the timing of differentiation at molecular and cytochemical levels and found that in all culture conditions full development of these cells was obtained, although at different times. The spontaneous differentiating potential of these cells strongly suggests their possible applications in regenerative medicine.
\end{abstract}

Keywords: Dental pulp stem cells, melanocytes, differentiation, melanosomes, L-Dopa.

*Address for correspondence:

Virginia Tirino or Gianpaolo Papaccio

Department of Experimental Medicine,

Histology and Embryology,

Tissue Engineering and Regenerative Medicine (TERM)

Division

School of Medicine, Second University of Naples,

5 via L. Armanni,

I-80138 Napoli, Italy

Telephone Number: +390815667715

FAX Number: +390815667720

E-mail: virginia.tirino@unina2.it or gianpaolo.papaccio@unina2.it

\section{Introduction}

Dental pulp stem cells (DPSCs) are multipotent stem cells derived from both neural crest and mesenchyme, therefore displaying a variety of characteristics as well as differentiation potentials.

Several studies have been carried out on these cells which have demonstrated that they include more than one cell population (Kerkis et al., 2006; Sloan and Waddington, 2009; Waddington et al., 2009). In fact, two different stem cell populations have been identified: i) a neural crest-derived cell population, expressing the lowaffinity nerve growth factor receptor (LANGFR), which is an embryonic neural crest cell marker, and ii) a mesenchymal cell population, expressing the $\beta 1$-integrin receptor subunit (Waddington et al., 2009). Both have the ability to differentiate into osteoblasts, adipocytes, and chondrocytes (Waddington et al., 2009). Although the majority of the studies have focused their attention on the ability of DPSCs to differentiate into odontoblast-like cells (Almushayt et al., 2006; Cordeiro et al., 2008; Paula-Silva et al., 2009) or osteoblasts (Laino et al., 2005; Laino et al., 2006; D'Aquino et al., 2007; Otaki et al., 2007), it is also known that they are also capable of differentiating into other cell types, including smooth muscle cells (Kerkis et al., 2006; D’Aquino et al., 2007; Gandia et al., 2008) and neurons (Arthur et al., 2008; Kadar et al., 2009). In addition, it has been shown that only the CD34- DPSC subpopulation is capable of differentiating into melanocyte-like cells when cultured in a specific melanogenic medium (Stevens et al., 2008).

Both melanocytes and dental pulp cells arise from the neural crest cell population. These cells migrate from the dorsal neural tube and undergo an epithelial-mesenchymal transition during embryonic development (Dupin and Le Douarin, 2003). They stop their migration at different anatomical sites, giving rise to various differentiated cell types, including neurons and glial cells of the peripheral nervous system, endocrine cells, some skeletal elements, tendons and smooth muscle, chondrocytes, osteocytes, and melanocytes. In particular, in the head and neck, neural crest cells, known as CNCCs (cranial neural crest cells), form the craniofacial mesenchyme and contribute to tooth morphogenesis (Thesleff and Sharpe, 1997; Han et al., 2003; Dupin et al., 2007) through a complex cross-talk with the oral epithelium, giving rise to condensed dental mesenchyme, dental papilla, odontoblasts, dentine matrix, cementum, periodontal ligaments, and dental pulp (Chai et al., 2000). 
Therefore, we can suppose that melanocytes and dental pulp tissue have a common ancestral progenitor.

Melanocytes are elongated dendritic cells with specific organelles, called melanosomes, which contain all components required for melanin biosynthesis. The main melanosomal proteins involved as catalytic and/or structural components in melanin biosynthesis include tyrosinase (TYR), the tyrosinase-related proteins-1 (TRP1) and TRP-2 (Hearing and Tsukamoto, 1991; Tsukamoto et al., 1992; Kwon, 1993), gp100 (Kwon et al., 1991; Kobayashi et al., 1994; Zhou et al., 1994), and MART-1 (Melanoma Antigen Recognized by T lymphocytes), a protein required for melanosome structure and maturation (Kawakami et al., 1994; Kawakami et al., 1997; Hoashi et al., 2005) .

In the present study we focused our attention on the melanocyte differentiation capacity of DPSCs and showed that these cells express the above mentioned proteins involved in the melanogenesis process, at different times during their differentiation in vitro. Interestingly, DPSCs express TRP-2, TRP-1, gp100/Pmel and MART-1 antigens even when they are not stimulated by the selective differentiating medium for melanocytes. Furthermore, we demonstrated that DPSCs differentiate into mature melanocytes; in fact, these cells contain melanosomes at the terminal stage of maturation.

Our findings demonstrate for the first time that dental pulp stem cells are capable of spontaneously differentiating into fully mature melanocytes without any culture stimulation, highlighting that these cells are excellent candidates for cell therapy and regenerative medicine in melanocyte-associated diseases.

\section{Materials and Methods}

\section{Cell culture and media}

Human dental pulp was extracted from molars of healthy subjects, after informed consent. Dental pulp tissue was gently separated from the crown and root and then digested in a solution of $3 \mathrm{mg} / \mathrm{ml}$ collagenase type I and $4 \mathrm{mg} / \mathrm{ml}$ dispase for 1 hour at $37^{\circ} \mathrm{C}$. Single-cell suspensions were obtained by passing the cells through a $70 \mu \mathrm{m}$ Falcon strainers (Becton \& Dickinson, Sunnyvale, CA, USA).
After this filtration, cells cultures were established by seeding single-cell suspensions into T-25 flasks in MegaCell $^{\mathrm{TM}}$ Minimum Essential Medium Eagle (Sigma, St. Louis, MO, USA), supplemented with $10 \%$ foetal bovine serum (FBS), $100 \mu \mathrm{M}$ L-ascorbic acid 2-phosphate, $2 \mathrm{mM}$ L-glutamine, $100 \mathrm{U} / \mathrm{ml}$ penicillin, and $100 \mu \mathrm{g} / \mathrm{ml}$ streptomycin (Invitrogen, Carlsbad, CA, USA). The cells were incubated at $37^{\circ} \mathrm{C}$ in $5 \% \mathrm{CO}_{2}$. After two passages in this control medium, the cells were grown in specific culture medium to induce melanocyte differentiation.

Melanocyte differentiation medium was composed of DMEM (Sigma), 10\% FBS, supplemented with $1.5 \mu \mathrm{g} / \mathrm{ml}$ hydrocortisone (Sigma), $10 \mu \mathrm{g} / \mathrm{ml}$ insulin (Sigma), $10 \mu \mathrm{g} /$ $\mathrm{ml}$ transferrin and, daily, $4 \mathrm{ng} / \mathrm{ml}$ basic Fibroblast Growth Factor (bFGF) (Invitrogen). Moreover, cells were cultured in Dulbecco's Modified Eagle's Medium (DMEM) supplemented with $10 \%$ FBS as a vehicle control. Cells were grown for more than 120 days. The three different culture media were changed twice a week.

Cells were passaged at the time of the experimental assay or when at confluence.

Cultures and assays described below were all performed in quadruplicate.

\section{RNA extraction and RT-PCR}

Total RNA was extracted using TRIzol Reagent (Invitrogen, Life Technologies) according to the manufacturer's protocol. RNA concentration and purity were determined using a UV spectrophotometer by $\mathrm{A}_{260}$ and $A_{260} / A_{280}$ ratio, respectively. The integrity of total RNA was assessed on standard $1 \%$ agarose/formaldehyde gels.

DNase treatment was performed to eliminate any residual genomic DNA. cDNA was obtained from $1 \mu \mathrm{g}$ of total RNA using reverse transcriptase (Promega, Madison, WI, USA) and random primers (Promega) in a final volume of $20 \mu \mathrm{l}$. Real-time polymerase chain reactions (RT-PCRs) were carried out using the primer sequences listed in Table 1. Thermal cycle parameters were: $95^{\circ} \mathrm{C}$ for $2 \mathrm{~min}, 40$ cycles of $95^{\circ} \mathrm{C}$ for $30 \mathrm{~s}, 60^{\circ} \mathrm{C}$ for $1 \mathrm{~min}$ and $72^{\circ} \mathrm{C}$ for $30 \mathrm{~s}$. The RT-PCR products were separated on $2 \%$ agarose gel electrophoresis, stained with ethidium bromide, and photographed under UV illumination.

These assays were performed only for qualitative purposes.

Table 1. Primer sequences and product sizes used for RT-PCR.

\begin{tabular}{llc}
\hline Gene & \multicolumn{1}{c}{ Sequence (5'-3') } & Product size (bp) \\
\hline MART-1 F & GATCATCGGGACAGCAAAGT & 137 \\
$\boldsymbol{M A R T - 1 \boldsymbol { R }}$ & AGGTGTCTCGCTGGCTCTTA & \\
$\mathbf{g p 1 0 0} \mathbf{F}$ & AAGGTCCAGATGCCAGCTCAATCA & 187 \\
$\mathbf{g p 1 0 0} \mathbf{R}$ & AGGATCTCGGCACTTTCAATACCC & \\
$\boldsymbol{T R P - 1} \boldsymbol{F}$ & TGTCCTCCTGCACACCTTCACA & 325 \\
$\boldsymbol{T R P - 1} \boldsymbol{R}$ & ATCCATACTGCGTCTGGCACGA & \\
$\boldsymbol{T R P - 2} \boldsymbol{F}$ & GAAACCACCAGTGATTCGGCA & 383 \\
$\boldsymbol{T R P - 2} \boldsymbol{R}$ & CAGAGTCGTTGGCTGTGAA & \\
\hline
\end{tabular}

F- forward; $R$ - reverse 
Table 2. Table showing the percentage of MART-1 and L-DOPA positive cells.

\begin{tabular}{|c|c|c|c|}
\multicolumn{1}{c}{} & \multicolumn{1}{c}{$\begin{array}{c}\text { \% MART-1 } \\
\text { positive cells }\end{array}$} & \multicolumn{1}{c}{$\begin{array}{c}\text { \% L-DOPA } \\
\text { positive cells }\end{array}$} \\
\hline Culture medium & $\mathbf{1 5 0}$ days & $\mathbf{1 5 0}$ days & $\mathbf{1 8 0}$ days \\
\hline MEM & $22.7 \pm 1.9$ & 0 & $12.5 \pm 0.6$ \\
\hline DMEM & $43.2 \pm 4.4^{*}$ & $21.7 \pm 1.5$ & $\begin{array}{c}32.6 \pm \\
2.7 * *\end{array}$ \\
\hline
\end{tabular}

Data are given as mean \pm SD. ${ }^{*} p<0.01 v s$. MEM 150 days; $* * p<0.01$ vs. MEM 180 days and DMEM 150 days.

\section{Immunohistochemical analysis}

Immunohistochemistry for MART-1 (Sigma) was performed on cells cultured in 24 well plates, fixed with $4 \%$ paraformaldehyde for $10 \mathrm{~min}$ at $4^{\circ} \mathrm{C}$ and rinsed in PBS. For staining, the DAKO Cytomation En Vision+HRP kit AEC (DAKO, Milan, Italy) was used according to the manufacturer's instructions. The MART-1 antibody was diluted 1:50 in PBS. Cells were observed using an inverted light microscope.

In order to quantify the percentage of Mart-1 positive cells, 20 fields, in each slide, were randomly selected from four independent experiments, then analysed by two independent researchers, counting the number of Mart-1 positive cells with respect to the total cell numbers. Results were analyzed with $t$ test using the Excel 2003 (Microsoft, Redmond, WA, USA) software program. Data are shown as mean $\pm \mathrm{SD}$ in Table 2. $P$ values $<0.05$ were considered significant.

\section{Flow cytometry and cell sorting}

Cells were detached using trypsin-EDTA in phosphatebuffered saline (PBS), counted and washed in $0.1 \%$ BSA in PBS. At least 500,000 cells were incubated with mouse anti-human CD34 PE conjugated (Miltenyi Biotec S.r.1. Calderara di Reno, Bologna, Italy) for $30 \mathrm{~min}$ at $4^{\circ} \mathrm{C}$ in the dark. After washing steps, the labelled cells were analyzed by flow cytometry using a FACS AriaII (Becton $\&$ Dickinson, Mountain View, CA, USA) and then sorted for CD34. The purity of sorted populations was routinely $90 \%$. Isotype antibodies were used as controls.

\section{Immunofluorescence analysis}

Dental pulp cells were plated in 2 glass chamber-slides and cultured for 2 days. Cells were rinsed twice with PBS and fixed with $4 \%$ paraformaldehyde in PBS for $10 \mathrm{~min}$ at $4^{\circ} \mathrm{C}$. After washing twice with PBS, the cells were permeabilised with $0.1 \%$ Triton X-100 in PBS for 5 min. at room temperature. Then they were washed twice with PBS, and incubated in PBS containing 5\% bovine serum albumin (BSA) for $30 \mathrm{~min}$. at room temperature as a blocking step. The cells were then incubated in 1\% BSA/ PBS containing MART-1, overnight at $4{ }^{\circ} \mathrm{C}$ in the dark.

This was followed by incubation with the secondary antibody FITC-conjugates. Nuclear counterstaining was performed with 4,6-diamidino-2-phenylindole (DAPI). After extensive washing with PBS, coverslips were mounted onto slides. Stained cells were observed using an inverted confocal laser scanning microscope (LSM 510 Met, Zeiss, Göttingen, Germany).

\section{Transmission electron microscopy}

Human dental pulp cells cultured as described above were fixed in $2.5 \%$ glutaraldehyde in $0.1 \mathrm{M}$ cacodylate buffer (pH 7.4), postfixed in $1 \% \mathrm{OsO}_{4}$ in Zetterquist buffer, dehydrated in ethanol, and embedded in epoxy resin. Ultrathin sections were contrasted in aqueous uranylacetate and lead-hydroxide, studied and photographed by a Hitachi 7000 Transmission Electron Microscope (Hitachi, Tokyo, Japan).

\section{L-DOPA assay}

Cells were stained using the L-DOPA reaction for tyrosinase activity. L-Dopa (3,4- dihydroxyphenylalanine) has been shown to be a key substrate of tyrosinase (Slominski et al., 1988). Cells were rinsed twice in $0.1 \mathrm{M}$ sodium phosphate buffer $\mathrm{pH} 6.8$, fixed for $20 \mathrm{~min}$ in $4 \%$ paraformaldehyde, washed three times with phosphate buffer and incubated at $37^{\circ} \mathrm{C}$ for up to $18 \mathrm{~h}$ in the dark with $5 \mathrm{mM}$ L-DOPA (Sigma) in $0.1 \mathrm{M}$ sodium phosphate buffer, $\mathrm{pH}$ 6.8. After incubation, the cells were rinsed with distilled water, fixed for $20 \mathrm{~min}$ in 4\% paraformaldehyde, and photographed by light microscopy.

In order to quantify the percentage of L-DOPA positive cells, 20 fields, in each slide, were randomly selected from four independent experiments, then analysed by two independent researchers, counting the number of L-DOPA positive cells with respect to the total cell numbers. Results were analyzed with $t$ test using the Excel 2003 software program. Data are shown as the mean \pm SD in Table 2. $P$ values $<0.05$ were considered significant.

\section{Statistical evaluation}

Student's $t$-test was used for statistical evaluation. A $p<0.05$ was considered significant.

\section{Results}

\section{DPSC cultures and morphology}

Cells were cultured under three different conditions (see Materials and Methods section for details): i) in Mega Cell Minimum Essential Medium Eagle (MEM), used as the standard medium; ii) in Melanocyte Differentiating Medium (MDM), and iii) in DMEM that was used as a control for the MDM medium.

No differences in size or shape among cells cultured in MDM, DMEM, and MEM were detected. DPSCs were characterized by a typical fibroblast-like morphology, spindle-shaped with long cytoplasmic processes, and tended to align themselves in parallel lines (Fig. 1). Dental pulp cells were harvested and analyzed at different culture times $(30,60,90,120,150$, and 180 days).

\section{Temporal gene expression during melanocyte differentiation of DPSCs}

In order to assess the melanocyte differentiation of DPSCs, we first performed RT-PCR for MART-1, gp100, TRP-1, and TRP-2 (Fig. 2). After 30 and 60 days of culture, cells grown in MEM, in DMEM, and in MDM expressed only gp100. At 90 days, RT-PCR analysis revealed TRP-2 
expression, in addition to gp100 expression, in cells cultured in MDM and in DMEM, while there was no TRP2 expression in cells cultured in MEM. Interestingly, after 120 days in all three culture conditions cells expressed TRP-1 and TRP-2. At this time, MART-1 gene was expressed only in cells grown in MDM and in DMEM (Fig. 2A), while, after 5 months (about 150 days) of culture, it was also expressed in cells cultured in MEM (Fig. 2B).

Moreover, in order to investigate which subpopulation of dental pulp was capable of differentiating into melanocytes, CD34- antigen expressing cells were sorted with a FACS ARIA II at the beginning of the culture. RTPCR analysis on these cells showed that CD34 ${ }^{+}$and CD34" cells both expressed MART-1 (Fig. 2C) at the same culture times described above.

Because cells cultured in MDM showed the same morphological features and pattern of antigenic expression as cells cultured in DMEM, we decided to consider only MEM and DMEM cell cultures for our further investigations.

\section{Immunocytochemical analysis to assess the presence of MART-1 protein}

In order to verify if MART-1 messenger RNA was effectively translated inside the cells, immunocytochemical analyses were carried out. We observed that MART-1 protein was expressed in a perinuclear localization both in cells grown in MEM and in those grown in DMEM after 150 days of culture (Fig. 3A,B), and that MART-1 staining appeared weaker in cells cultured in MEM than in DMEM. The same distribution pattern of MART-1 was detected in melanocytes cultured as positive control. (Fig. $3 \mathrm{C})$. The percentages of positive cells are shown in Table 2.

\section{Subcellular characterization of MART-1 antigen by confocal microscopy}

After 180 days of culture we performed confocal microscopy study to better characterize the subcellular localization of MART-1 in DPSCs (Fig.4). This analysis revealed two different subtypes in MART-1 positive cells cultured in DMEM: i) one displaying a granular staining that was more intense in the perinuclear region (showing a distribution pattern similar to those previously observed and described) (Fig. 4B); and ii) another displaying fine granular structures diffused toward the cell periphery (Fig. 4C). At the same culture time in MEM cultured cells, discrete MART-1 positive punctate staining was evident throughout the cytoplasm (Fig. 4A). These MART-1 distribution patterns are compatible with the presence of melanosomes in MEM- and in DMEM-cultured cells.

\section{Ultrastructural analysis of DPSCs}

Aiming to demonstrate the formation of melanosomes in the cytoplasm of DPSCs cultured in MEM and in DMEM, we carried out transmission electron microscopy (TEM) analyses. TEM observations revealed the presence of melanosomes at four different stages of development (Fig.5, bottom panel) in cells grown in MEM (Fig. 5A,B) and in DMEM (Fig. 5C,D) after 180 days of culture. During
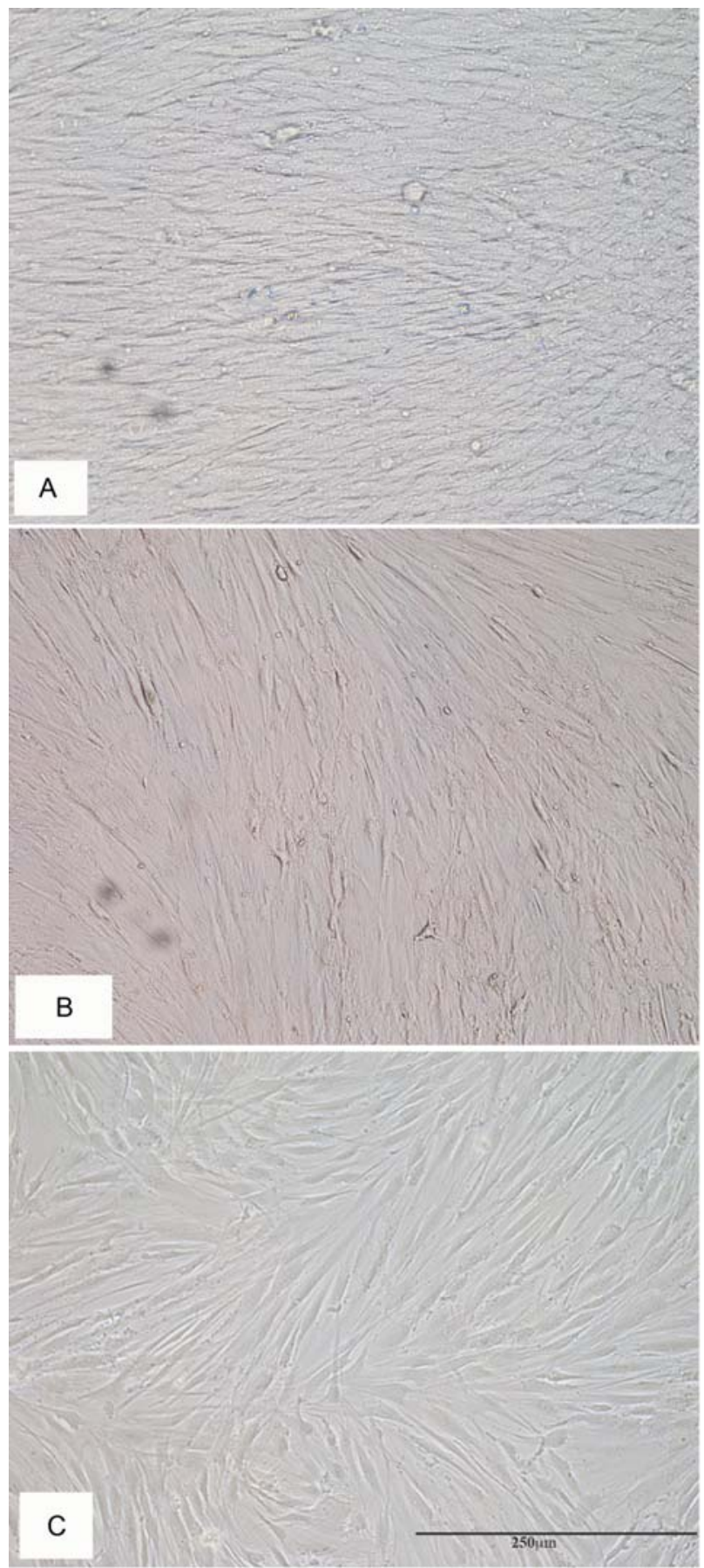

Fig. 1. Morphology of DPSCs cultured in different media. (A) DPSCs cells cultured in MEM, (B) in DMEM and $(\mathbf{C})$ in MDM media. In all three conditions cells display a fibroblast-like morphology with elongated cytoplasmic processes arranged in parallel orientation.

stage I, melanosomes were spherical organelles with intraluminal vesicles lacking melanin; at stage II, they had an elliptical shape and contained a gray, striated, fibrillar internal matrix; at stage III, the striated fibrillar matrix became more electron-dense, clearly indicating melanin deposition; and at stage IV, the organelles were completely filled with melanin (Fig. 5, bottom panel). We noted that stage III melanosomes were the most frequently observable. In addition, we did not find melanosomes in 
A
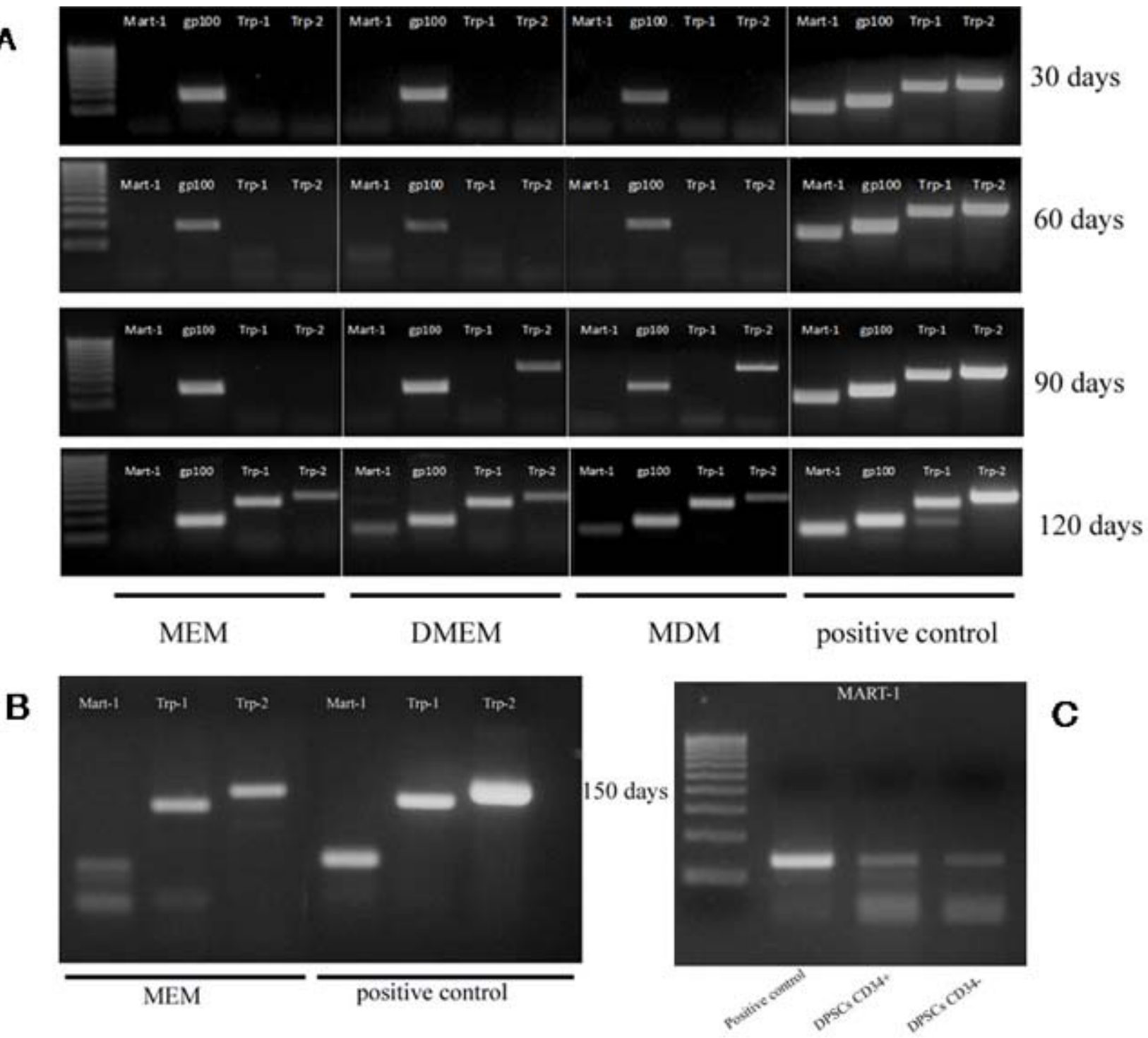

Fig. 2. Melanocyte gene expressions at different time of DPSCs differentiation. (A) RT-PCR analysis of gp100, Trp-1, Trp-2 and MART-1 at 30, 60, 90 and 120 days of culture of DPSCs cultured in MEM, DMEM and MDM. (B) RT-PCR analysis of MART-1, Trp-1 and Trp-2 in MEM cultured cells at 150 days of culture. (C) RT-PCR analysis for MART-1 on CD34+ and CD34- DPSCs subpopulations.

cells grown in MEM up to 120 days of culture (data not shown).

\section{L-DOPA assay for functional analysis in vitro of the melanocyte-differentiated DPSCs}

In order to assess the activity of tyrosinase, which is the enzyme involved in melanin biosynthesis, we performed L-DOPA assays. The results showed that at 150 days of culture, cells in MEM were negative for tyrosinase activity (Fig. 6A) while those cultured in DMEM were positive (Fig. 6B). The percentages of positive cells are shown in Table 2.

Interestingly, as the cell culture time progressed (about 180 days) the cells cultured in MEM became positive for the DOPA assay (Fig. 6C), demonstrating that the differentiated melanocytes acquired the tyrosinase activity for melanin biosynthesis although at different times of culture, depending on the medium used.

\section{Discussion}

Dental pulp stem cells have a great potential in regenerative medicine because of their capacity to differentiate into several different types of cells: osteoblasts, adipocytes, chondrocytes, odontoblasts, neurons, and also melanocytes (Laino et al., 2005; Almushayt et al., 2006; D'Aquino et al., 2007; Otaki et al., 2007; Arthur et al., 2008; Cordeiro et al., 2008; Gandia et al., 2008; Stevens et al., 2008; Kadar et al., 2009; Paula-Silva et al., 2009). In addition, their capacity to repair human bone defect when combined with appropriate scaffolds, in the same patient has been recently demonstrated (D'Aquino et al., 2009). This study aimed to assess the melanocytic differentiation potential of human DPSCs in vitro, comparing lineage-specific differentiation medium with basal media. We demonstrated that human DPSCs, which are normally fated to the osteoblasticlineage (Laino et al., 2005; d'Aquino et al., 2007; Otaki et 


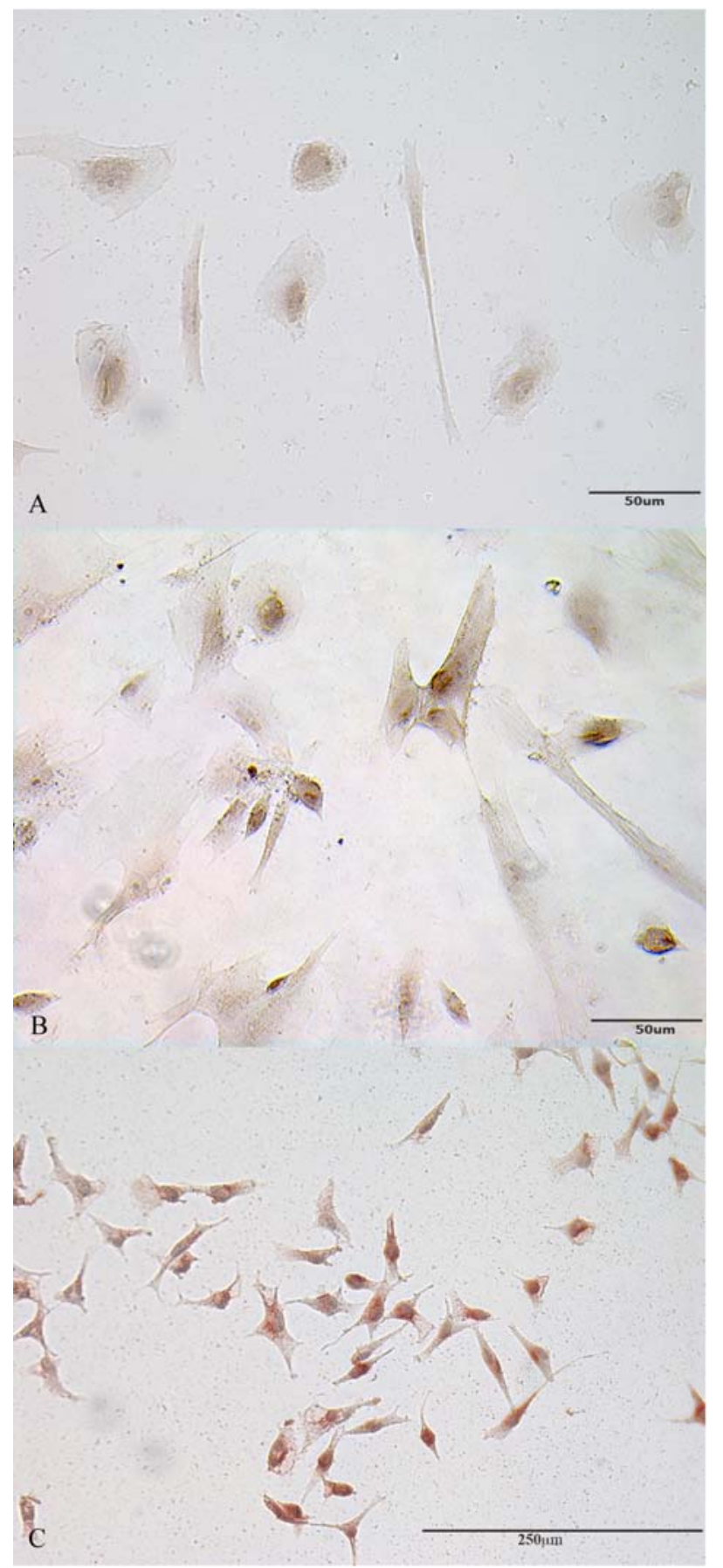

al., 2007), also retain an intrinsic capacity to become melanocytes, and that this tendency is considerably enhanced when they are exposed to specific culture conditions. This spontaneous differentiation into melanocytes may be ascribed to the fact that DPSCs, originating from the neural crest-like melanocytes, somehow still retain neural crest properties.

For this reason, we propose two hypotheses for the melanocyte differentiation of DPSCs: either the DPSC population still contains neural crest cells that undergo a switch to the melanocyte-lineage in vitro, or whole dental pulp contains melanocyte precursors in a quiescent state (or in the G0/G1 phase of cell cycle), which are activated to proliferate in vitro and, thus, become quantitatively detectable. Both these hypotheses are supported by the RT-PCR analyses reported in this study. In fact, our results at the early passages we did not detect melanocytic markers, while after 90 days of culture, TRP-2 was expressed in cells grown in DMEM and in MDM media, indicating the beginning of melanocyte differentiation. TRP-2 antigen, indeed, is considered an early melanoblast marker that is expressed much earlier than TRP-1 during melanocyte development (Steel et al., 1992; Hornyak, 2006). In this context, gp100 expression was not considered because, according to Brouwenstijn et al. (1997), we found that this marker was transcripted in a lot of cell lines of non-melanocytic origin that we used as negative controls.

As the cell cultures progressed to 120 days, DPSCs grown in DMEM and in MDM media expressed all the melanocyte-specific markers that we considered (MART1, Trp-1, and Trp-2), meaning that melanocyte differentiation progressed in these cultures, although at the same culture time, cells grown in MEM did not express MART-1. The expression of this marker in cells grown in MEM was observed only later, at 150 days of culture,

Fig. 3. Immunocytochemical analysis for MART-1 antigen. The analysis shows a weak positive stain in MEM culture (A) and a more evident stain in cells cultured in DMEM, with perinuclear distribution (B). The same distribution pattern was detected in melanocyte cells used as positive control (C).

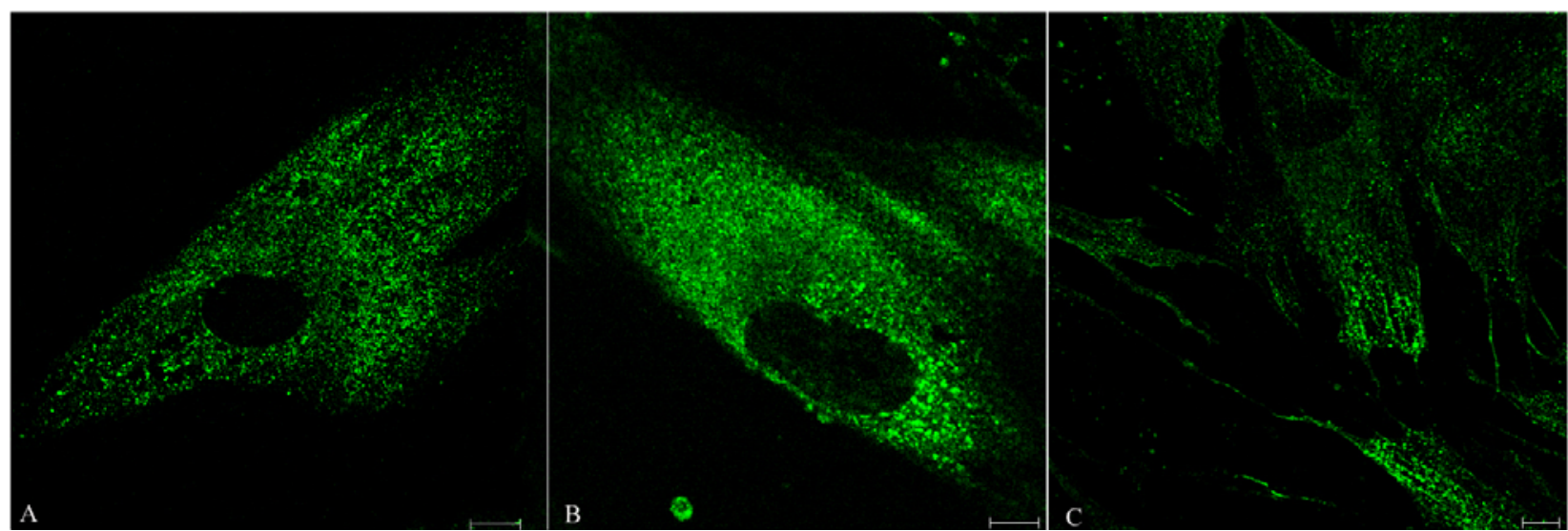

Fig. 4. Subcellular localisation of MART-1 examined by confocal microscopy. Cells exhibited a granular staining pattern with a cytoplasmic distribution in MEM cultured cells (A) and a perinuclear localization (B) or toward the cell periphery (C) in DMEM cultured cells. Scale bar: $10 \mu \mathrm{m}$ 

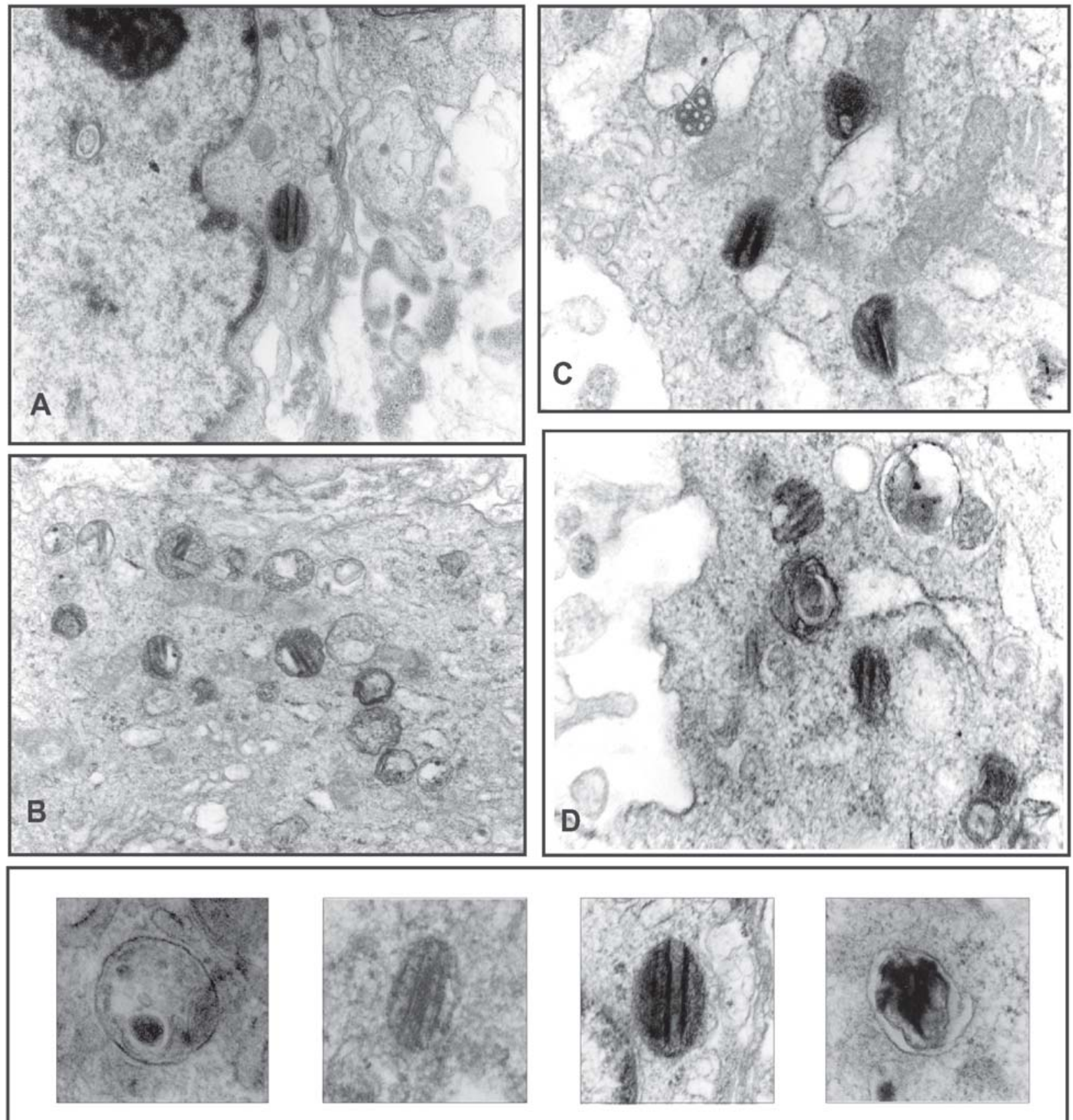

।

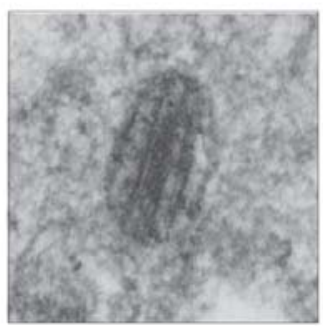

II

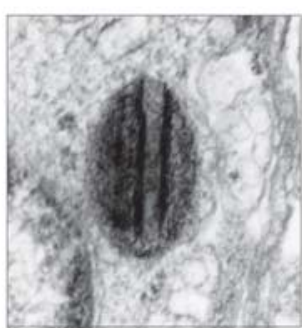

III

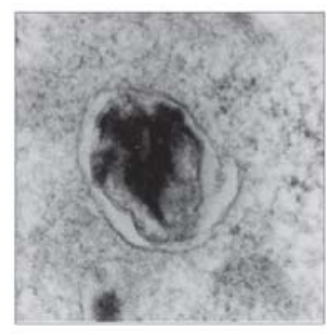

IV

Fig. 5. Ultrastructural analysis of cultured DPSCs. Transmission electron microscopy after 180 days of culture of MEM cultured cells (A-B) and DMEM cultured cells (C-D). Scale bar: $1 \mu \mathrm{m}$. In the bottom panel the four different stages (I-IV) of melanosome development found in both culture conditions are reported. Scale bar: $0.5 \mu \mathrm{m}$.

indicating that the melanocyte differentiation in this control medium occurs spontaneously, but slower.

Moreover, we found that DMEM enhanced differentiation. In fact, the cells grown in DMEM expressed the MART-1 melanocyte marker earlier than the cells cultured in MEM. This may due to the fact that the culture medium DMEM is rich in L-tyrosine, which is necessary for melanogenesis (Smit et al., 1997; Slominski et al., 1999). Furthermore, in order to investigate which subpopulation of dental pulp stem cells was capable of differentiating into melanocytes, we performed cell sorting for the CD34 antigen. We considered the CD34 antigen because it has been reported by some authors that DPSCs belong to the $\mathrm{CD} 34^{+}$subpopulation (Cordeiro et al., 2008), while by others to the CD34 one (Stevens et al., 2008; Agha-Hosseini et al., 2009). We noted that both the sorted CD34+ and CD34- fractions of dental pulp cells expressed the specific melanocyte marker MART-1. This suggests that in DPSCs, the precursors with melanocyte differentiation ability may lie upstream of the CD34 positive cell population.

Because it has been reported that the MART-1 antigen is restricted to cells of the melanocytic lineage (Rimoldi et al., 2001; Hoashi et al., 2005), we focused our attention 


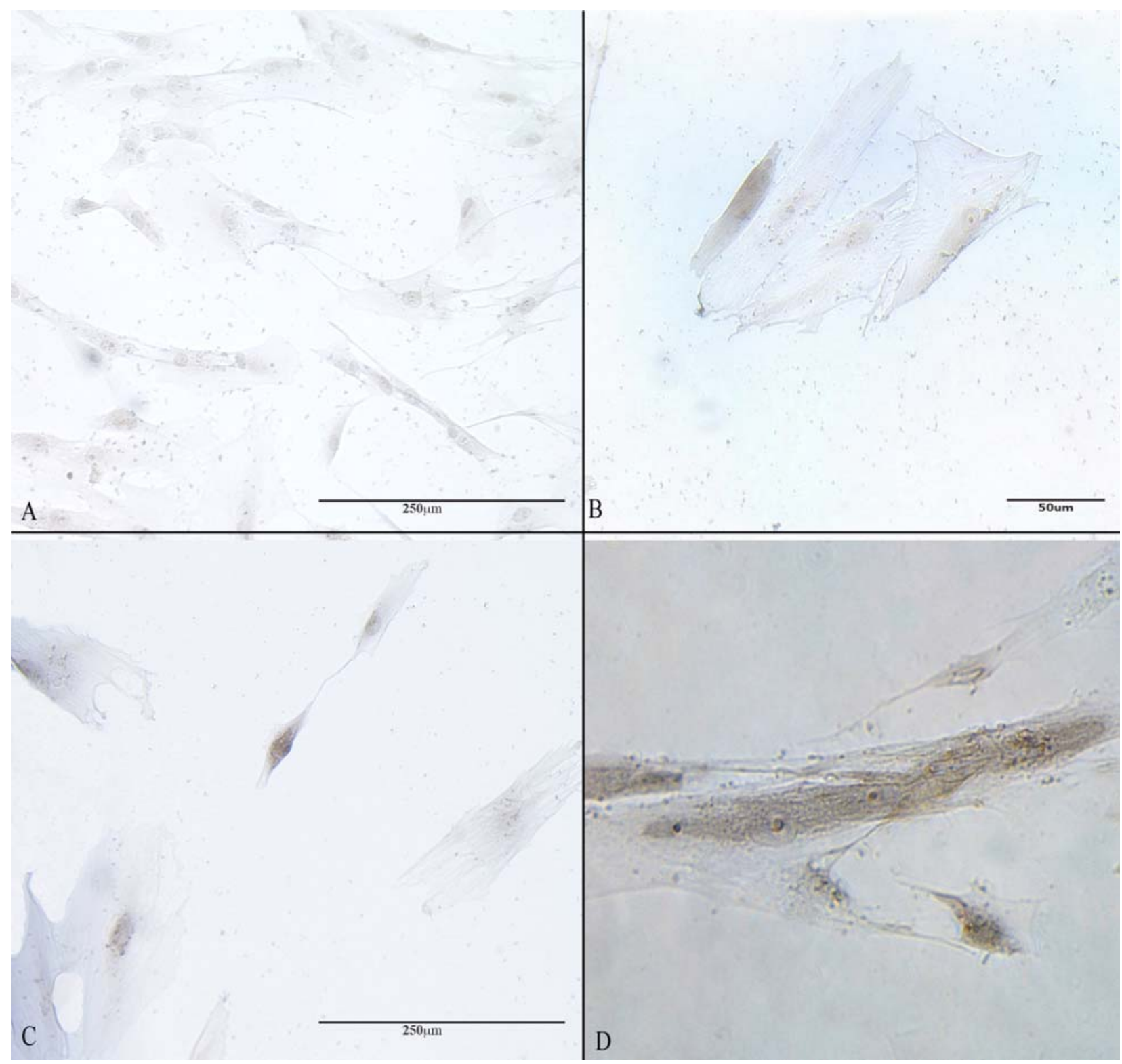

Fig. 6. Tyrosinase (dopa-oxidase) activity in DPSCs. (A) DOPA-negative staining of DPSCs grown in MEM compared with (B) DOPA-positive staining of DPSCs grown in DMEM at 150 days of culture. After 180 days, DOPApositive cells were also present in MEM (C). A detail of DOPA-positive cells in DMEM culture (D).

only on this melanocyte-specific marker for the next investigations.

At 150 days of culture, we performed an immunocytochemical analysis that revealed the presence of the MART-1 antigen with a perinuclear distribution pattern in all three cell culture conditions. It has previously been reported that the MART-1 antigen has a perinuclear localization in human melanoblast cell cultures (Cook et al., 2003) and that it is present in large amounts on the surface of melanosomes, especially in their early stages of maturation (De Maziere et al., 2002). On the basis of these previously reported data we can assume that in our DPSCs at 150 days of culture melanocyte precursors (melanoblasts) were present.

In addition, we performed confocal microscope analyses 30 days after the immunocytochemical analysis (180 days of culture) to better characterize the subcellular localization, and we were able to demonstrate discrete MART-1 staining points with a variable distribution pattern dependent on the culture conditions: from the perinuclear area, granular staining was present throughout the cytoplasm (in MEM cell culture) or diffused towards the cell periphery (in DMEM cell culture). This distribution pattern is compatible with physiological melanosome maturation and consequently with melanoblast differentiation into melanocytes. Melanosomes are specific organelles of melanocytes in which melanin is synthesized and stored (Kushimoto et al., 2001; Kushimoto et al., 2003). During their maturation, melanosomes move from the perinuclear area towards the plasma membrane. They often are divided into early melanosomes (stages I e II), generally found in the Golgi area (Kushimoto et al., 2003), and in late melanosomes (stages III and IV), which are transported toward the cell periphery (Watabe et al., 2008). 
On the basis of these observations, we carried out transmission electron microscopy (TEM) analyses to be sure that melanosomes were present in the cytoplasm of DPSCs cultured in either MEM or DMEM. TEM analyses confirmed our hypotheses, highlighting the presence of melanosomes at the four different stages of maturation (IIV), based on morphology and melanin content (Raposo et al., 2001; Seiji et al., 1963); actually, we observed that stage III was the most frequent in DPSCs. Moreover, TEM analyses did not reveal melanosome structures up to 120 days of culture.

In order to confirm that the melanosomes reached the final stage of differentiation and that DPSCs were differentiated into functionally active melanocytes, we performed L-DOPA assays. L-DOPA is used as the substrate for the DOPA oxidase activity of tyrosinase, the key enzyme in melanin biosynthesis (Hearing and Ekel, 1976). The L-DOPA assay detected tyrosinase activity in cells grown in DMEM at 150 days of culture and later (at 180 days) in cells cultured in MEM, according to our previous experiments.

\section{Conclusions}

This study shows for the first time that: i) DPSCs spontaneously differentiate in vitro toward the melanocytic lineage; ii) these cells show morphological and molecular features of melanocytes; iii) DPSCs cultured in MEM differentiate into melanocytes considerably slower than those cultured in DMEM. In addition, we demonstrated that DPSCs, other than exhibiting a spontaneous melanocytic potential, are capable of forming fully differentiated melanosomes and, therefore, functionally active melanocytes.

The novelty of this study leads us to suggest that DPSCs might be used for therapeutic purposes in various diseases caused by defective pigment cells, such as vitiligo vulgaris, an acquired pigmentary disorder of the skin characterized by circumscribed depigmented macules and patches in which some or all of the melanocytes are selectively destroyed, or in reconstructive surgery after severe burns that frequently heal with pigmentary changes and/or permanent depigmentation of the skin. The use of DPSCs for this purpose is proposed because these cells are a resource that requires non-invasive or minimally invasive collection procedures. Usually, non permanent teeth naturally exfoliate without trauma and, during life, almost all persons undergo third molar extraction due to disodontiasis. This is of rather importance, taking also into consideration that it is usually extremely difficult to collect human samples for stem cell use and, in particular, the use of DPSCs to obtain melanocytes, will avoid that large skin samples should be removed from patients for grafting into depigmented areas. These cells, therefore, could be used for all the above purposes without great manipulation in vitro because they spontaneously differentiate into melanocytes, as we have shown in this study.

\section{Acknowledgements}

The authors thank Dr. Gaetano Calì at the IEOS "G. Salvatore", National Council of Research, Naples, Italy for confocal microscope assistance and the Department of Histology and Medical Embryology, University of Rome "La Sapienza", for TEM facilities. Dr. Rachel Sammons (University of Birmingham, Edgbaston, Birmingham, B15 2TT, UK) and Michael VG Latronico, ITB, CNR, Milan (Italy) are kindly acknowledged for English editing.

This study was supported by SIRIO-FIRB 2007/2009 grant n. 06 n. RBIP06FH7J_006 to G.Pa.

\section{References}

Agha-Hosseini F, Jahani MA, Jahani M, MirzaiiDizgah I, Ali-Moghaddam K (2009) In vitro isolation of stem cells derived from human dental pulp. Clin Transplant 24: E23-28.

Almushayt A, Narayanan K, Zaki AE, George A (2006) Dentin matrix protein 1 induces cytodifferentiation of dental pulp stem cells into odontoblasts. Gene Ther 13: 611-620.

Arthur A, Rychkov G, Shi S, Koblar SA, Gronthos S (2008) Adult human dental pulp stem cells differentiate toward functionally active neurons under appropriate environmental cues. Stem Cells 26: 1787-1795.

Brouwenstijn N, Slager EH, Bakker AB, Schreurs MW, Van der Spek CW, Adema GJ, Schrier PI, Figdor CG (1997) Transcription of the gene encoding melanomaassociated antigen gp100 in tissues and cell lines other than those of the melanocytic lineage. Br J Cancer 76: 1562-1566.

Chai Y, Jiang X, Ito Y, Bringas P, Jr., Han J, Rowitch DH, Soriano P, McMahon AP, Sucov HM (2000) Fate of the mammalian cranial neural crest during tooth and mandibular morphogenesis. Development 127: 1671-1679.

Cook AL, Donatien PD, Smith AG, Murphy M, Jones MK, Herlyn M, Bennett DC, Leonard JH, Sturm RA (2003) Human melanoblasts in culture: expression of BRN2 and synergistic regulation by fibroblast growth factor-2, stem cell factor, and endothelin-3. J Invest Dermatol 121: 11501159.

Cordeiro MM, Dong Z, Kaneko T, Zhang Z, Miyazawa M, Shi S, Smith AJ, Nor JE (2008) Dental pulp tissue engineering with stem cells from exfoliated deciduous teeth. J Endod 34: 962-969.

D’Aquino R, Graziano A, Sampaolesi M, Laino G, Pirozzi G, De Rosa A, Papaccio G (2007) Human postnatal dental pulp cells co-differentiate into osteoblasts and endotheliocytes: a pivotal synergy leading to adult bone tissue formation. Cell Death Differ 14: 1162-1171.

D’Aquino R, De Rosa A, Lanza V, Tirino V, Laino L, Graziano A, Desiderio V, Laino G, Papaccio G (2009) Human mandible bone defect repair by the grafting of dental pulp stem/progenitor cells and collagen sponge biocomplexes. Eur Cell Mater 18: 75-83. 
De Maziere AM, Muehlethaler K, van Donselaar E, Salvi S, Davoust J, Cerottini JC, Levy F, Slot JW, Rimoldi D (2002) The melanocytic protein Melan-A/MART-1 has a subcellular localization distinct from typical melanosomal proteins. Traffic 3: 678-693.

Dupin E, Le Douarin NM (2003) Development of melanocyte precursors from the vertebrate neural crest. Oncogene 22: 3016-3023.

Dupin E, Calloni G, Real C, Goncalves-Trentin A, Le Douarin NM (2007) Neural crest progenitors and stem cells. C R Biol 330: 521-529.

Gandia C, Arminan A, Garcia-Verdugo JM, Lledo E, Ruiz A, Minana MD, Sanchez-Torrijos J, Paya R, Mirabet V, Carbonell-Uberos F, Llop M, Montero JA, Sepulveda P (2008) Human dental pulp stem cells improve left ventricular function, induce angiogenesis, and reduce infarct size in rats with acute myocardial infarction. Stem Cells 26: 638-645.

Han J, Ito Y, Yeo JY, Sucov HM, Maas R, Chai Y (2003) Cranial neural crest-derived mesenchymal proliferation is regulated by Msx1-mediated p19(INK4d) expression during odontogenesis. Dev Biol 261: 183-196.

Hearing VJ, Ekel TM (1976) Mammalian tyrosinase. A comparison of tyrosine hydroxylation and melanin formation. Biochem J 157: 549-557.

Hearing VJ, Tsukamoto K (1991) Enzymatic control of pigmentation in mammals. FASEB J 5: 2902-2909.

Hoashi T, Watabe H, Muller J, Yamaguchi Y, Vieira WD, Hearing VJ (2005) MART-1 is required for the function of the melanosomal matrix protein PMEL17/ GP100 and the maturation of melanosomes. J Biol Chem 280: 14006-14016.

Hornyak TJ (2006) The developmental biology of melanocytes and its application to understanding human congenital disorders of pigmentation. Adv Dermatol 22: 201-218.

Kadar K, Kiraly M, Porcsalmy B, Molnar B, Racz GZ, Blazsek J, Kallo K, Szabo EL, Gera I, Gerber G, Varga G (2009) Differentiation potential of stem cells from human dental origin - promise for tissue engineering. J Physiol Pharmacol 60 Suppl 7: 167-175.

Kawakami Y, Eliyahu S, Delgado CH, Robbins PF, Rivoltini L, Topalian SL, Miki T, Rosenberg SA (1994) Cloning of the gene coding for a shared human melanoma antigen recognized by autologous $\mathrm{T}$ cells infiltrating into tumor. Proc Natl Acad Sci U S A 91: 3515-3519.

Kawakami Y, Battles JK, Kobayashi T, Ennis W, Wang X, Tupesis JP, Marincola FM, Robbins PF, Hearing VJ, Gonda MA, Rosenberg SA (1997) Production of recombinant MART-1 proteins and specific antiMART-1 polyclonal and monoclonal antibodies: use in the characterization of the human melanoma antigen MART1. J Immunol Methods 202: 13-25.

Kerkis I, Kerkis A, Dozortsev D, Stukart-Parsons GC, Gomes Massironi SM, Pereira LV, Caplan AI, Cerruti HF (2006) Isolation and characterization of a population of immature dental pulp stem cells expressing OCT-4 and other embryonic stem cell markers. Cells Tissues Organs 184: $105-116$.

Kobayashi T, Urabe K, Orlow SJ, Higashi K, Imokawa G, Kwon BS, Potterf B, Hearing VJ (1994) The Pmel 17/ silver locus protein. Characterization and investigation of its melanogenic function. J Biol Chem 269: 29198-29205.

Kushimoto T, Basrur V, Valencia J, Matsunaga J, Vieira WD, Ferrans VJ, Muller J, Appella E, Hearing VJ (2001) A model for melanosome biogenesis based on the purification and analysis of early melanosomes. Proc Natl Acad Sci U S A 98: 10698-10703.

Kushimoto T, Valencia JC, Costin GE, Toyofuku K, Watabe H, Yasumoto K, Rouzaud F, Vieira WD, Hearing VJ (2003) The Seiji memorial lecture: the melanosome: an ideal model to study cellular differentiation. Pigment Cell Res 16: 237-244.

Kwon BS (1993) Pigmentation genes: the tyrosinase gene family and the pmel 17 gene family. J Invest Dermatol 100: 134S-140S.

Kwon BS, Chintamaneni C, Kozak CA, Copeland NG, Gilbert DJ, Jenkins N, Barton D, Francke U, Kobayashi Y, Kim KK (1991) A melanocyte-specific gene, Pmel 17, maps near the silver coat color locus on mouse chromosome 10 and is in a syntenic region on human chromosome 12. Proc Natl Acad Sci U S A 88: 9228-9232.

Laino G, d'Aquino R, Graziano A, Lanza V, Carinci F, Naro F, Pirozzi G, Papaccio G (2005) A new population of human adult dental pulp stem cells: a useful source of living autologous fibrous bone tissue (LAB). J Bone Miner Res 20: 1394-1402.

Laino G, Carinci F, Graziano A, d'Aquino R, Lanza V, De Rosa A, Gombos F, Caruso F, Guida L, Rullo R, Menditti D, Papaccio G (2006) In vitro bone production using stem cells derived from human dental pulp. J Craniofac Surg 17: 511-515.

Otaki S, Ueshima S, Shiraishi K, Sugiyama K, Hamada S, Yorimoto M, Matsuo O (2007) Mesenchymal progenitor cells in adult human dental pulp and their ability to form bone when transplanted into immunocompromised mice. Cell Biol Int 31: 1191-1197.

Paula-Silva FW, Ghosh A, Silva LA, Kapila YL (2009) TNF-alpha promotes an odontoblastic phenotype in dental pulp cells. J Dent Res 88: 339-344.

Raposo G, Tenza D, Murphy DM, Berson JF, Marks MS (2001) Distinct protein sorting and localization to premelanosomes, melanosomes, and lysosomes in pigmented melanocytic cells. J Cell Biol 152: 809-824.

Rimoldi D, Muehlethaler K, Salvi S, Valmori D, Romero P, Cerottini JC, Levy F (2001) Subcellular localization of the melanoma-associated protein MelanAMART-1 influences the processing of its HLA-A2restricted epitope. J Biol Chem 276: 43189-43196.

Seiji M, Fitzpatrick TB, Simpson RT, Birbeck MS (1963) Chemical composition and terminology of specialized organelles (melanosomes and melanin granules) in mammalian melanocytes. Nature 197: 10821084.

Sloan AJ, Waddington RJ (2009) Dental pulp stem cells: what, where, how? Int J Paediatr Dent 19: 61-70.

Slominski A, Moellmann G, Kuklinska E, Bomirski A, Pawelek J (1988) Positive regulation of melanin pigmentation by two key substrates of the melanogenic pathway, L-tyrosine and L-dopa. J Cell Sci 89: 287-296.

Slominski A, Ermak G, Wortsman J (1999) Modification of melanogenesis in cultured human 
melanoma cells. In Vitro Cell Dev Biol Anim 35: 564 565 .

Smit NP, Van der Meulen H, Koerten HK, Kolb RM, Mommaas AM, Lentjes EG, Pavel S (1997) Melanogenesis in cultured melanocytes can be substantially influenced by L-tyrosine and L-cysteine. J Invest Dermatol 109: 796800 .

Steel KP, Davidson DR, Jackson IJ (1992) TRP-2/DT, a new early melanoblast marker, shows that steel growth factor (c-kit ligand) is a survival factor. Development 115: 1111-1119.

Stevens A, Zuliani T, Olejnik C, LeRoy H, Obriot H, Kerr-Conte J, Formstecher P, Bailliez Y, Polakowska RR (2008) Human dental pulp stem cells differentiate into neural crest-derived melanocytes and have label-retaining and sphere-forming abilities. Stem Cells Dev 17: 11751184.

Thesleff I, Sharpe P (1997) Signalling networks regulating dental development. Mech Dev 67: 111-123.

Tsukamoto K, Jackson IJ, Urabe K, Montague PM, Hearing VJ (1992) A second tyrosinase-related protein, TRP-2, is a melanogenic enzyme termed DOPAchrome tautomerase. EMBO J 11: 519-526.

Waddington RJ, Youde SJ, Lee CP, Sloan AJ (2009) Isolation of distinct progenitor stem cell populations from dental pulp. Cells Tissues Organs 189: 268-274.

Watabe H, Valencia JC, Le Pape E, Yamaguchi Y, Nakamura M, Rouzaud F, Hoashi T, Kawa Y, Mizoguchi M, Hearing VJ (2008) Involvement of dynein and spectrin with early melanosome transport and melanosomal protein trafficking. J Invest Dermatol 128: 162-174.

Zhou BK, Kobayashi T, Donatien PD, Bennett DC, Hearing VJ, Orlow SJ (1994) Identification of a melanosomal matrix protein encoded by the murine si (silver) locus using "organelle scanning". Proc Natl Acad Sci U S A 91: 7076-7080.

\section{Discussion with Reviewer}

Reviewer III: The authors indicate that these cells may provide appropriate tools to heal melanocyte-related diseases. These diseases are very limited in number and constitute only minor danger for the patient's health. Skin pigmentation is not a crucial problem, and the answer that might be provided is more related to the field of aesthetics rather than to a health problem. Is it worth to extract a sound tooth to get access to a pulp and/or keep cultured pulp cells in a cell bank? Other more abundant sources of melanocytes may be found. Please comment.

Authors: We agree that Vitiligo disease is not painful, contagious, or fatal. The death of melanocytes may leave a person with a stigmatized appearance which is considered only a "cosmetic disorder", but this pigment loss may negatively affect the patient's quality of life by causing a great deal of emotional pain. The patient may feel depressed by cosmetic disfigurement, anxious about the spread of vitiligo. Furthermore, the juvenile patient with vitiligo may need special attention, especially the junior high-school age child. There is a considerable body of research suggesting that people with vitiligo have a greater risk of depression, anxiety, and suicidal thoughts. Although approximately one-third of the patients do not appear to suffer negative psychological effects from vitiligo and appear to adjust well to the disorder, two-thirds manifest some degree of shame or other negative psychological effects (e.g., Sampogna et al., 2008; additional reference). Therefore, we think that these psychological effects are not negligible.

There are various treatments for vitiligo, e.g., monochromatic excimer light (MEL), Polypodium leucotomos extract and pimecrolimus cream but these do not show impressive results. Autologous melanocyte transplantation is the most advanced and latest surgical treatment: after anaesthetising the healthy skin, usually from thigh or buttocks, a small part of the skin is removed, cultured or not, and may then be grafted onto the areas of vitiligo. This is, however, an invasive technique to obtain melanocytes and often scars or de-pigmentation occur in the donor areas, whereas with our proposal teeth could be used: it is common to lose teeth without trauma.

\section{Additional Reference}

Sampogna F, Raskovic D, Guerra L, Pedicelli C, Tabolli S, Leoni L, Alessandroni L, Abeni D (2008) Identification of categories at risk for high quality of life impairment in patients with vitiligo. Br J Dermatol 159: 351-359. 